\title{
REFLEXIONS SUR LES CHANGEMENTS INDUITS PAR LE NUMERIQUE DANS L'ENSEIGNEMENT ET L'APPRENTISSAGE DES LANGUES ${ }^{1}$
}

À chaque époque ses changements et ses manières d'en rendre compte ; des positionnements s'expriment sur fond de querelle des anciens et des modernes dans laquelle les uns voient le retour du même, suscitant les mêmes débats autour d'objets fétichisés (autrefois l'audiovisuel, l'autonomie, aujourd'hui le numérique..), alors que les autres parient sur des modifications qualitatives des pratiques d'enseignants et d'apprenants de langues ainsi que des recherches qui y sont associées. Nous prenons clairement position ici pour le second parti, quitte à sous-estimer certains aspects relevant de la pérennité de l'enseignement/ apprentissage des langues, mais dans l'optique de rendre visibles certains traits qui peuvent ne pas apparaître à ceux qui enseignent les langues sans s'intéresser aux TIC.

Il peut toutefois paraître déraisonnable de chercher à réfléchir sur les changements survenus dans le domaine de la didactique des langues sous l'effet de la montée du numérique dans notre paysage contemporain. D'une part, le recul nécessaire pour pouvoir juger des pratiques et comportements stabilisés (le seront-ils jamais ?) n'est pas suffisant, une quinzaine d'années seulement, d'autre part, les changements en termes d'outils de communication toujours plus nombreux et rapides, d'années en années rendent par avance dépassés au moment de leur lecture les écrits tentant de rendre compte des nouveautés en la matière. Pour autant, tant l'enseignement que l'apprentissage des langues changent (c'est ce que l'on va chercher à montrer), et il apparaît nécessaire de chercher à en rendre compte ne serait-ce que pour prendre date par rapport à l'histoire de la discipline, souligner la rapidité avec laquelle ces changements se sont opérés et pour pointer les questions que soulèvent, tant au niveau des pratiques que de la recherche, les nouveaux contextes numériques. Nous reprenons l'acronyme TIC pour parler des outils numériques en général, étant entendu que nous ne nous livrerons pas à un état de l'art concernant tel ou tel support ${ }^{2}$.

Nous nous proposons plutôt d'interroger l'articulation entre les TIC et le domaine de l'enseignement/ apprentissage des langues à partir d'une première question : quels changements les TIC ont-elles apportés tout d'abord à notre rapport aux langues, au savoir en général, puis à l'enseignement/apprentissage des langues sur le plan des pratiques des enseignants et des apprenants ?

Cette première question se situe donc sur un plan général, au niveau macro : quels sont les changements liés à l'épistémologie numérique ? La seconde partie s'appuiera sur un exemple précis, sur le plan méso d'une formation de futurs enseignants de FLE à l'université. Dans cet article, nous parlerons indifféremment de «numérique», de «TIC» ou de

\footnotetext{
${ }^{1}$ L'auteur remercie Christelle Celik, Tatiana Codreanu, Anthippi Potolia et Viorica Nicolaev pour leurs suggestions et leurs relectures qui ont permis d'améliorer une première version de cet article.

${ }^{2}$ A l'image de celui proposé par RoberVanderplank sur les laboratoires de langues, la télévision et la vidéo (2009).
} 
« technologies» sachant que ces termes renvoient surtout pour nous aux contraintes et opportunités induites par leur utilisation suivant déjà sur ce point Benkler : « Ni déterministe, ni totalement malléable, la technologie définit certains paramètres de l'action individuelle et sociale. Elle peut ainsi faciliter ou compliquer la mise en place de certaines actions, relations, organisations et institutions. " (Benkler, 2006 : 48). Nous aurons ainsi, à plusieurs reprises au fil de ces lignes, recours à la figure du paradoxe ou de la tension entre forces antagonistes pour présenter différents aspects de la situation que nous cherchons à décrire. Nous avons choisi d'adopter une perspective " historique à court terme » en nous référant à la fin des années 90- début des années 2000 pour illustrer l'écart entre les idées circulant à cette époque et celles d'aujourd'hui en prenant comme cadre de réflexion les travaux de Colin Lankshear (2003) concernant ce qu'il nomme « l'épistémologie numérique ».

\section{Les changements liés à l'épistémologie numérique}

Colin Lankshear a identifié quatre types de changements liés à la révolution des technologies de l'information (Castells, 2001) : «les changements dans le monde à connaître, dans la conception du savoir et les processus d'accès au savoir, dans la nature de ceux qui savent et dans l'importance relative des différents modes du savoir $»^{3}$. Ce philosophe de l'éducation s'intéresse aux changements que le numérique a entraînés dans la vie sociale en général alors que nous allons, en ce qui nous concerne, nous focaliser sur le domaine de l'enseignement/apprentissage des langues. $C^{\prime}$ 'est à partir de cette déclinaison des changements proposée par Lankshear que nous chercherons à spécifier certains changements intervenus et à voir sous quels aspects ils ont pu avoir une influence sur les pratiques et discours des enseignants et des apprenants, en nous en tenant à chaque fois à un ou deux exemples. Attachons-nous tout d'abord aux changements dans le monde à connaître.

\section{Changements dans le monde à connaître}

Il est éclairant de se reporter ne serait-ce qu'une dizaine d'années en arrière, lors de la percée d'Internet dans la vie sociale, pour se remémorer les interrogations que suscitait l'examen des sites de l'époque chez les chercheurs linguistes en particulier. Ainsi, dans une journée organisée par le CREDIF en mai 2000, Monique Slodzian faisait observer que « la révolution numérique met [tait] les langues au défi : lesquelles seront demain réellement présentes dans la compétition ouverte par les nouveaux outils de l'information et de la commutation? » et s'interrogeait sur « le sort des langues qui ne pourront pas participer à la création de contenus culturels et de savoirs nouveaux, largement diffusés sur les nouveaux réseaux, (qui) devront faire face à de nouveaux périls. ». Elle pensait que « la compétition se jouant désormais dans le pré carré de la culture et des savoir-faire technologiques, rien d'étonnant que la très grande majorité des langues du monde soient en difficulté, voire condamnées à un déclin aussi rapide

\footnotetext{
${ }^{3}$ Notre traduction.
} 
qu'inéluctable » (2000 : 137-138). Il était, en effet, à l'époque, assez peu imaginable qu'une encyclopédie en ligne comme Wikipédia propose, en 2011, des articles dans plus de 270 langues et soit même, dans une langue comme le kiswahili ${ }^{4}$, la première encyclopédie existante, précédant toute autre sur quelque support que ce soit. Si jamais exposition à tant de langues n'a été aussi facile qu'aujourd'hui, cela ne veut pas dire que l'appropriation, du savoir se fait aisément. Nous nous plaçons ici au niveau du monde à connaître, et pas au niveau des processus à mettre en œuvre pour accéder à la connaissance. Reconnaissons cependant qu'Internet a facilité l'accès à la variété linguistique contrairement à ce que l'on pouvait craindre au départ, puisque même s'il est majoritairement anglophone, de nombreuses autres langues ont trouvé moyen d'y trouver place à l'exemple du site Wikipédia dans lequel la langue anglaise représente moins de $20 \%$ des articles.

Pour en venir au domaine plus spécifique de l'apprentissage des langues, dès l'apparition du multimédia dans les années 90, les chercheurs en didactique des langues (Barbot, 1997 ; Develotte, 1997) ont pointé les potentialités de ces supports, principalement, à l'époque, des cédéroms, en termes d'apprentissage des langues: en effet, la multimodalité (ou coprésence du son, du texte, de l'image fixe et de la vidéo), liée à l'interactivité et à l'hypertextualité permettaient d'envisager une vision renouvelée de l'apprentissage des langues. Ces différentes modalités, activables au gré de l'utilisateur et dissociables entre elles, ont donné naissance, par exemple, à des méthodes qui proposaient une progression graduelle dans l'accès à la compréhension d'une situation. Ces supports conçus pour une utilisation individuelle (et non plus en classe entière sous la direction de l'enseignant), ont ainsi donné la possibilité de visionner (autant de fois que souhaité) une vidéo présentant l'interaction entre deux locuteurs français puis d'activer dans l'ordre et au moment voulus, une série d'aides ${ }^{5}$. Ce processus d'aide graduée, associé à la gestion individuelle constituait une des originalités de ces supports et les rendaient particulièrement intéressants dans une optique d'auto-apprentissage. En outre, cette même multimodalité les rendaient aptes à proposer des apprentissages différenciés puisqu'ils sollicitaient de façon séparée ou simultanée, l'ouïe, la vue, le geste graphique et permettaient donc aux concepteurs de jouer sur différentes entrées susceptibles de convenir à des profils d'apprenants diversifiés (Barbot, 1997). La généralisation du haut débit a ensuite ouvert les possibilités de communication et de nouvelles perspectives dans l'enseignement/apprentissages des langues, en synchronie en particulier, sur lesquelles nous reviendrons.

\section{Changements dans la conception du savoir et les processus d'apprentissage}

Au cours des dix dernières années, c'est dans le cadre de ce que l'on

\footnotetext{
${ }^{4}$ Les premiers 25000 articles dans cette langue sont en ligne (entretien de Jimmy Wales, Digital Planet, BBC 19 janvier 2011).

${ }^{5}$ Par exemple : la présentation écrite des mots clés expliqués, la transcription en français de chaque phrase, voire sa traduction dans la langue de l'apprenant.
} 
appelle le $\mathrm{Web} 2.0^{6}$ que se sont opérées les innovations dans l'apprentissage et l'enseignement des langues. Les changements les plus notables sont liés aux différents procédés et dispositifs de mise en contact des locuteurs entre eux, sur lesquels nous allons revenir plus loin. Mais il ne faudrait pas penser que seules les technologies ont permis le développement de nouvelles pratiques. En fait, c'est tout le contexte scientifico-économico-politicosocial qui a conduit à ces modifications en didactique des langues. Parmi les facteurs importants de changements, on trouve le développement socioéconomique de ce que l'on appelle la mondialisation, l'accroissement des mobilités et échanges internationaux (par exemple, la construction politique de l'Europe), l'utilité grandissante de la maîtrise des langues, à l'oral en particulier dans de nombreux secteurs de la vie professionnelle. Sur le plan scientifico-institutionnel, les travaux du Conseil de l'Europe ont fait figurer les compétences de production orale en interaction (différenciée de la production orale monologuée) parmi les compétences de l'apprenant de langue.

Au niveau de la didactique des langues, si l'approche communicative cherchait, déjà à partir de 1970 à précisément travailler cette compétence de production de discours en interaction, elle avait largement recours à des simulations ou à des jeux de rôles pour mettre les apprenants en situation de communiquer entre eux. L'approche actionnelle développée plus récemment (Cadre européen commun de référence, 2000) se fonde sur l'articulation des situations d'apprentissage avec les situations sociales. Les apprenants de LE sont ainsi incités à tester leurs compétences dans le cadre d'activités suscitant des feed-backs sociaux : que ce soit pas le biais des medias sociaux (hors institution) ou dans le cadre plus formel de la classe par des appariements d'apprenants, la mise en contact des personnes entre elles entre donc tout à fait dans cette perspective actionnelle. On voit donc que les possibilités de mise en contact liées aux TIC sont venues se greffer dans un contexte prêt à les accueillir et à les exploiter sous différentes formes. À travers différents types d'appariements, en mode synchrone et/ou asynchrone, il est désormais possible d'apprendre une langue étrangère en ligne si tant est que l'on soit doté d'un ordinateur et d'une connexion à haut débit (ce qui est, soulignons-le, loin d'être le cas, partout dans le monde...).

Des partenariats peuvent s'effectuer sur le modèle du projet Tandem $^{7}$, avec des enseignants (de nombreuses entreprises commerciales en ligne existent déjà sur ce créneau) voire de futurs enseignants comme par exemple, le dispositif «le français en (première) ligne $»^{8}$.

C'est sur cette dernière formation, sur laquelle nous reviendrons plus en détail en deuxième partie, que je m'arrêterai un instant pour illustrer les changements de processus d'apprentissage produits par un dispositif d'enseignement/apprentissage en ligne particulier. Actuellement, les échanges en ligne entre futurs enseignants et apprenants de langue

\footnotetext{
${ }^{6}$ Ce terme désigne certaines technologies du Web en particulier les interfaces permettant aux internautes d'interagir (partager, échanger, etc.) de façon simple, à la fois avec le contenu et la structure des pages.

${ }^{7} \mathrm{http}: / /$ www.cisi.unito.it/tandem/email/idxeng00.html

${ }^{8}$ http://w3.u-grenoble3.fr/fle-1-ligne/index.html
} 
s'effectuent à partir de la plate-forme $V i s u^{9}$ en cours de développement à Lyon. Conçu à partir des analyses d'usage effectuées sur les premières années d'utilisation de $M S N$ et de Skype, cet outil a été développé en trois espaces (salon de préparation, salon d'interactions, salon de rétrospection), en différenciant l'écran de l'apprenant de celui de l'enseignant et en prévoyant l'enregistrement des séances d'interactions synchrones. Dans cette interface de communication qui a donc été créée pour répondre tant aux besoins d'un apprenant de langues qu'à ceux d'un enseignant en ligne synchrone, les participants ont la possibilité, lors de leurs échanges dans le salon d'interactions, de poser des marqueurs qui signalent des moments de l'interaction sur lesquels ils pourront revenir, soit à la fin de l'exercice ou de la séance, soit après coup, de façon asynchrone. Si l'on se place du côté de l'enseignant, les marqueurs peuvent concerner des problèmes de prononciation, de syntaxe, de lexique... Sans interrompre le cours de l'interaction, l'enseignant peut donc signaler (ces marques ne sont pas visibles sur l'écran de l'apprenant pour ne pas être inhibantes), les points susceptibles d'amélioration par la suite. Symétriquement, l'apprenant peut utiliser des marqueurs (ces derniers étant visibles par l'enseignant) pour signaler par exemple, qu'il ne comprend pas, qu'il faut répéter, etc. L'enregistrement des interactions permet aux participants de revenir $a$ posteriori sur les échanges : l'enseignant en ligne peut compléter, dans le salon de rétrospection, le feed-back qu'il souhaite adresser à l'apprenant (soit par écrit, soit par oral, en enregistrant la bonne prononciation d'un mot par exemple à la suite de l'imperfection qui a été notée). De même l'apprenant peut se réécouter et reprendre, en les répétant, les améliorations qui lui ont été suggérées par le tuteur.

On voit dans ce cas de figure que tant « la conception du savoir» que « les processus d'accès au savoir » sont modifiés en fonction de la nature spécifique de l'apprentissage revisité par les potentialités liées non seulement aux fonctionnalités techniques développées (enregistrement, marqueurs, etc.) mais aussi au traitement cognitif qui y est associé. $\mathrm{Si}$, par exemple, l'enseignant est en train de parler et que l'apprenant éprouve des difficultés de compréhension, au lieu d'avoir à interrompre son interlocuteur ou, plus poliment, d'attendre qu'il s'arrête de parler, l'apprenant peut indiquer par un marqueur ${ }^{10}$, dans le fil du discours oral de l'enseignant que celui-ci parle trop vite permettant la régulation des interactions au fur et à mesure que les difficultés se font ressentir.

L'apprentissage des langues se trouve ainsi reconfiguré par ces nouvelles conditions matérielles, il est donc nécessaire de prendre la mesure du type d'apprentissage le plus adapté à ces dispositifs numériques. Et ce sont différents types de compétences qui sont alors développées selon que l'on interagit sur une base principalement textuelle (à partir d'un forum et de messages échangés en asynchronie) ou plutôt orale via une plate-forme

\footnotetext{
${ }^{9}$ Plate-forme d'enseignement/apprentissage des langues développée par les universités de Lyon 1 et 2 et le TECFA de Genève dans le cadre de l'ANR Ithaca.

${ }^{10}$ Mettant également en œuvre ce que Jones (2004) nomme la polyfocalisation de 1'attention, c'est-à-dire la capacité à exécuter différentes choses en même temps, ici à la fois écouter et poser des marqueurs.
} 
audiographique (de type Lyceum) ou encore en visioconférence poste à poste (MSN, Skype). De nouveaux environnements d'apprentissage apparaissent par le biais des réseaux sociaux (de type Facebook) ou des mondes virtuels (Second life). L'interaction apprenant-enseignant dans des contextes médiés par des avatars ouvre par exemple de nombreux pans de recherche.

\section{Changements de ceux qui savent}

En 1979 Régis Debray stigmatisait ainsi le système de production concernant l'écrit: "Quarante médiocrates (au grand maximum) ont pouvoir de vie ou de mort sur quarante mille auteurs [...]. Pour les travaux des uns et des autres, ils constituent le sas à passage obligatoire séparant l'événement du non-événement, l'être du néant, l'utile de l'absurde ». (175-176). Si on peut penser que la situation n'a guère évolué depuis lors concernant les supports traditionnels, une brèche dans la liberté de diffusion s'est ouverte avec la publication en ligne. L'absence d'une instance légitimatrice des discours produits sur Internet change considérablement les contraintes d'écriture par les auteurs (de site, blog, wiki, etc.). Par ailleurs l'appareil de production atypique (qui est soumis à de très faibles contraintes économiques) fait voler en éclats le formatage traditionnel des écrits en fonction d'un marché de lecteurs précis. En termes de possibilités de création, on constate une ouverture : la diffusion publique du discours individuel est devenue possible et a permis à des productions hors normes de voir le jour sans être censurées par des instances de légitimation. De nombreux ouvrages, bandes dessinées, morceaux musicaux ont été édités à la suite du succès qu'ils avaient obtenu en première instance sur Internet.

Différents phénomènes ont eu une influence sur le changement survenu : le phénomène de crowdsourcing ${ }^{11}$ à savoir le fait qu' " en réunissant la plus grande quantité possible d'informations et d'opinions provenant de sources diverses, on obtient des résultats supérieurs à ceux que peuvent produire des experts. » (Pisani \& Piotet, $2008: 103$ ). Benkler souligne d'ailleurs l'extrême degré de sophistication qui peut être associé à l'évaluation de la pertinence des jugements des internautes ${ }^{12}$ dans le cas d'une création collaborative. Le poids de l'expert internaute est lié à l'avis des autres contributeurs eux-mêmes classés en fonction de la pertinence de leurs expertises. Le philosophe David Weinberger indique clairement ce changement: "Les experts ne sont plus les mêmes qu'avant. Maintenant, l'expert c'est tout le monde, comme le montre Wikipédia. Le savoir qui s'en dégage est souvent meilleur que celui que l'on aurait pu attendre d'un seul individu. L'expert ne disparaît pas mais on assiste à une sorte de négociation sociale du savoir. » (Pisani \& Piotet, 2008 : 116). C'est en tout cas un fait

\footnotetext{
${ }^{11}$ « La face créative et collaborative du crowdsourcing est bien réelle. Elle est la promesse d'un monde ouvert reposant sur le partage et la collaboration. Mais elle a son envers, plus inquiétant : l'opportunité qu'elle représente pour les plus malins $[\ldots]$ de faire travailler des volontaires sans avoir à les payer. » (Pisani : 140).

${ }^{12}$ Faisant suite au Manifeste des évidences, version française de Levine, R. Locke, C. Searls D. \& Weinberger, D., 1999, The Cluetrain Manifesto, http://www.cluetrain.com/manifeste.html )
} 
nouveau que les experts renvoient à ces sites collaboratifs plutôt qu'à euxmêmes quant à l'explicitation des termes qu'ils emploient. Ainsi Colin Lankshear et Michele Knobel dans la seconde édition de leur ouvrage New litteracies: everyday practices and classroom learning, avouent avoir renoncé à poursuivre la rédaction d'un glossaire après avoir vérifié que des sites tels que Wikipédia exprimaient mieux qu'eux-mêmes les définitions qu'ils auraient pu donner aux différentes notions et écrivent qu'ils préfèrent donc y renvoyer leurs lecteurs $(2010: 3)$.

Un autre phénomène est celui de l'influence des hackers dans la vie sociale : le récent scandale lié aux informations diffusées par Wikileaks en est une illustration : ceux qui savent aujourd'hui ne sont plus uniquement ceux qui étaient censés savoir. En fait, on s'aperçoit que c'est toute la sphère médiatique et communicationnelle qui modifie l'empan des destinataires d'une information, comme le montre le rôle qu'ont joué les médias sociaux dans les récentes révolutions arabes.

Enfin un autre phénomène est celui de la montée des licences libres, de l'open source qui s'illustre par exemple par le fait que des ouvrages majeurs soient à la fois accessible en ligne et sur papier (The Wealth of Networks, 2006, Access to Knowledge in the Age of Intellectual Property, 2010) et permettent une meilleure diffusion des idées et une plus grande rapidité de cette diffusion.

Dans un tel contexte, quels changements peut-on noter dans le domaine de l'enseignement et de l'apprentissage des langues? Actuellement, en particulier dans l'enseignement secondaire, les premières enquêtes effectuées (Guichon, à paraître) montrent que peu d'enseignants de langue utilisent les outils numériques. Chez les enseignants qui sont intéressés par les potentialités des outils numériques, on perçoit souvent une tension forte entre d'un côté leurs idées pédagogiques et d'autre part les contraintes institutionnelles dans lesquelles ils se trouvent. Lors du colloque Cyberlangues 2010 qui réunit, comme son nom l'indique, les enseignants de langues intéressés par les TIC, certains enseignants ont témoigné de leurs craintes à utiliser de façon régulière les TIC voire à montrer une séance d'enseignement utilisant les TIC lors des visites de leur inspecteur. En effet, ces visites sont rares (au maximum une tous les deux ans) et sont importantes car elles permettent à l'enseignant d'être évalué (et donc d'obtenir de possibles améliorations de traitement salarial). Or, la plupart des inspecteurs sont issus d'une culture d'enseignement traditionnelle et ne sont donc pas forcément ouverts à ce type de pratiques pour lesquels ils n'ont pas de critères d'évaluation définis. On voit dans cet exemple, que des enseignants, pourtant attirés et intéressés par les technologies sont en quelque sorte surdéterminés dans leurs pratiques, entre autres, par la hiérarchie dont ils dépendent et donc en proie aux tensions entre des forces antagonistes dont nous avons parlé en introduction.

\section{Changements dans la portée relative des différents modes de connaissance}

En 2003, une enquête relative aux pratiques de documentation des étudiants du département de français de l'université de Sydney mettait en 
évidence leurs utilisations déjà fréquentes d'Internet mais également les représentations associées au sérieux des différentes sources et à la confiance qu'on pouvait leur accorder (Develotte, 2003). Comme on peut s'y attendre, les livres étaient crédités d'une plus grande confiance que les sites Internet. Mais on peut supposer que la même enquête menée aujourd'hui donnerait des résultats différents : de nombreuses institutions légitimées ont pris place sur Internet, d'autres ont construit petit à petit leur légitimité comme Wikipédia, d'une part, par structuration interne et, d'autre part, par le soutien apporté par des institutions renommées (comme le British Museum par exemple) enfin, nous avons appris à vérifier les informations, à les recouper comme David Weinberger le note :

«L'information peut ne pas être exacte. Cependant sur l'Internet, on ne se borne pas à chercher de l'information, on essaie de mieux comprendre les choses que l'on sait déjà. Cette énorme pile de "choses", nous l'enrichissons avec autant de métadonnées que possible. De multiples façons (qu'il s'agisse de tags, des taxonomies d'hier, du web sémantique, de liens, de playlists ou de Digg) nous établissons des relations entre les choses, du sens. Nous ajoutons de la valeur. C'est le vrai web sémantique que nous créons, pas seulement pour savoir, mais pour comprendre. » (Pisani \& Piotet, 2008 : 116).

On voit donc que du point de vue cognitif, nous nous sommes adaptés à un univers de ressources dans lequel il faut trier, vérifier, valider par différents procédés les informations trouvées, ce qui nous renvoie aux changements dans les processus d'apprentissage évoqués précédemment.

Dans le domaine particulier de l'apprentissage des langues, un nouvel équilibre s'instaure entre l'apprentissage formel et informel: sous l'effet conjugué de l'intensification de la circulation des personnes et de la fréquentation des médias sociaux, les occasions d'exposition à des langues étrangères se sont multipliées. Des groupes d'intérêt concernant l'apprentissage des langues se sont constitués dans les médias sociaux (Xing, Facebook), des réseaux européens sont apparus : Language learning and social media : 6 key dialogues ${ }^{13}$ (coordonné par K. Zourou).

On constate ainsi que les affordances dynamiques (O'Rourke, 2005) de ces outils de communication ont permis qu'ils soient investis dans l'objectif précis d'apprentissage d'une langue étrangère ouvrant d'autant les possibilités d'accès par rapport à l'apprentissage classique en classe, par l'intermédiaire d'un professeur. Outre les outils de communication précédemment évoqués, d'autres vont également dans le sens d'une plus grande liberté octroyée à l'apprenant dans la gestion de son apprentissage. Il en va ainsi des plates-formes mobiles qui permettent de télécharger aussi bien des chansons que des émissions de radio ou tout autre document en langue étrangère et de les écouter au gré de ses trajets, (à pied ou dans les transports en commun), exploitant les potentialités de mobile learning offertes par ces objets miniaturisés. Dorénavant, on peut apprendre en bougeant, marchant, l'immobilité de la salle de classe n'est plus requise et la flexibilité s'exprime dans le terme ubiquitous learning.

13 http://www.elearningeuropa.info/languagelearning 


\section{Le numérique et l'enseignement-apprentissage du français à l'université}

C'est sur l'intuition que la mise en contact, via Internet, de locuteurs éloignés géographiquement était un levier potentiel de formation pour les futurs enseignants de français et à la fois de soutien à l'apprentissage de la langue pour les apprenants de français à l'étranger, que j'ai créé le projet Le français en (première) ligne (désormais $F 1 L$ ) lors de mon appartenance professionnelle au département de français de l'université de Sydney (Australie). Depuis neuf ans, le dispositif a évolué sous différentes formes et le nombre d'universités impliquées dans ce principe de partenariat interuniversitaires s'est multiplie ${ }^{14}$. De nombreuses publications ${ }^{15}$ sont en ligne sur le site du projet et nous nous contenterons ici de survoler les nouvelles compétences professionnelles de l'enseignant de langues que nous avons identifiées avant de nous intéresser aux recherches susceptibles d'être développées dans de tels contextes d'enseignement/apprentissage en ligne.

\section{De nouvelles compétences professionnelles pour l'enseignant}

$\mathrm{Au}$ départ conçu pour la communication asynchrone, via un forum ${ }^{16}$, il fonctionne depuis 2006 en communication synchrone entre les étudiants de master professionnel de l'université de Lyon $2^{17}$ et des apprenants de français de l'UC Berkeley ${ }^{18}$. Dans un récent article, F. Mangenot et moimême avons cherché à préciser les compétences professionnelles différentes auxquelles sont entraînés les futurs enseignants dans chacune des deux formations. Sans revenir sur ces savoir-faire, soulignons ici que les outils ont un effet sur la relation pédagogique et le développement d'un ethos d'enseignant particulier (2010b: 352) influencé par les affordances dynamiques des outils de communication. Les recherches menées autour de l'enseignement par visioconférence poste à poste, dans ce contexte situationnel précis ont ainsi permis d'identifier un genre de discours : la conversation pédagogique (Guichon, Drissi 2008) qui s'appuie donc sur les spécificités liées à ce type de communication (multimodale, hypertextuelle) et sur les aides technologiques telles que les marqueurs. Actuellement les formations de master FLE comportent souvent des modules liés aux TIC permettant ainsi une formation des futurs enseignants à plusieurs types d'enseignements (présentiels et en ligne).

Certains futurs enseignants sont ainsi formés aujourd'hui à enseigner selon différents formats (en présentiel et en ligne) et intègrent des «pluricompétences» professionnelles de façon concomitante. Les choses s'avèrent plus compliquées pour les enseignants déjà en exercice ${ }^{19}$. Il existe

\footnotetext{
${ }^{14}$ Site de l'AUF où les universités désireuses de mettre en place de telles interactions en ligne peuvent être mises en relation. http://www.aidenligne-francais-universite.auf.org/spip.php?page=sommaire appui ens fr

${ }^{15}$ Site du projet http://w3.u-grenoble3.fr/fle-1-ligne/publications.php

${ }^{16}$ associé au cours de master professionnel de François Mangenot

${ }^{17}$ Enseignants : Christine Develotte, C. \& Nicolas Guichon.

${ }^{18}$ Enseignants : Richard Kern \& Désirée Pries.

${ }^{19}$ Dans sa thèse, Ryogi Mogi (2010) explique que la capacité et le désir de changer chez les enseignants sont limités par
} 
en effet une tension entre des savoir-faire antérieurs, stabilisés (tels que ceux sur lesquels repose l'enseignement du français à un public étranger en classe) et des comportements fondés sur l'intuition, malléables, qui demandent à être travaillés pour en éprouver les apports et les limites. Ces nouvelles pratiques sont suscitées par l'intérêt des enseignants mais restent insécurisantes en ce sens que les enseignants sont amenés à tester les outils sans être assurés de leur fiabilité et en dehors de tout mode d'emploi. Alors que dans le cas de la classe d'apprenants, toute une économie de l'enseignement/apprentissage s'est structurée de longue date autour de manuels ou de la progression graduée prévue par l'institution, l'utilisation des TIC brouille les pistes : une multitude de possibilités d'ouverture de la classe vers l'extérieur (ou de « hors classe » complet) s'offrent maintenant. L'utilisation d'un Tableau Blanc Interactif (TBI), de blogs, des medias sociaux (Facebook, Twitter) sont de plus en plus intégrés par les enseignants dans des perspectives d'ouverture de la classe sur les ressources extérieures ou le maintien du contact hors classe (Poyet \& Develotte, 2011).

Il est nécessaire ici de ré-affirmer à ce propos le rôle crucial qui est celui de l'institution éducative dans l'enseignement des langues. En effet, dans un webinaire $^{20}$ intitulé "Social media and education : reasons to be fearful », diffusé le 9 février 2011 par l'université d'éducation de Londres, Nell Selwyn a enjoint ceux qui l'écoutaient à prêter attention à l'idéologie sousjacente aux médias sociaux. Pour lui, ces outils promeuvent l'individualisme et survalorisent la place de l'informel dans l'éducation. Ils risquent d'affaiblir l'éducation formelle dans sa vocation à construire des savoirs partagés et renvoient dans le domaine des pratiques privées des apprentissages traditionnellement pris en charge par l'institution. Ces craintes ne doivent pas être prises à la légère me semble-t-il et incitent à s'assurer que la pérennité d'un système dont tous les avantages ne sont peutêtre plus saillants, à force d'être banals, puisse être assurée.

D'où, la volonté de s'inscrire dans une démarche de consolidation des acquis de l'institution éducative plutôt que dans une entreprise de déstabilisation. Pour revenir sur le dispositif F1L illustrant cette partie, soulignons que les deux côtés (celui des futurs enseignants et celui des apprenants), l'enseignement s'effectue de façon "hybride» (blended learning) c'est-à-dire qu'il conjugue la communication en présentiel et la communication en ligne. Ce parti pris est crucial au niveau de l'équilibre à maintenir afin de structurer l'enseignement sur une base formelle et institutionnelle. De notre point de vue si l'école au sens large peut tirer profit à s'ouvrir à certaines utilisations des TIC, c'est toujours à partir d'un projet pédagogique qui précède l'utilisation des outils quels qu'ils soient.

\section{Le cadre des recherches sur l'enseignement/apprentissage des langues en ligne}

leur tendance à reproduire la culture d'enseignement (et d'apprentissage) qu'ils connaissent et qui sont à l'origine de leurs représentations de ce que sont l'enseignement et l'apprentissage.

${ }^{20}$ Séminaire se déroulant sur Internet, enregistré à l'adresse : http://cck11.mooc.ca/files/audio/cck11_09February11.mp3 . 
Nous venons de nous arrêter sur un dispositif de formation en ligne synchrone pour illustrer le tour que pouvait prendre tout à la fois la formation des enseignants et l'enseignement/apprentissage développés à travers elle. Les recherches associées à ce type de pratiques relèvent d'un spectre large dont le tableau ci-dessous cherche à rendre compte. Dans ce tableau, j'ai retenu comme discipline centrale les sciences du langage qui sont, en France, la discipline de référence pour la didactique des langues afin de montrer les différents niveaux de recherches qui doivent être développés. Figurent également les disciplines connexes qui peuvent être utilement convoquées dans le cadre d'un projet de recherche multi partenarial par exemple :

\begin{tabular}{|c|c|c|c|c|}
\hline & $\begin{array}{l}\text { Niveau de } \\
\text { recherche }\end{array}$ & $\begin{array}{l}\text { Corpus de } \\
\text { référence en } \\
\text { sciences du } \\
\text { langage }\end{array}$ & $\begin{array}{c}\text { Disciplines } \\
\text { connexes } \\
\text { convocables }\end{array}$ & $\begin{array}{l}\text { Questions } \\
\text { possibles }\end{array}$ \\
\hline Macro & $\begin{array}{l}\text { Ordre } \\
\text { économique } \\
\text { mondial/ } \\
\text { Education } \\
\text { nationale } \\
\text { Choix } \\
\text { politiques } \\
\text { institutionels }\end{array}$ & $\begin{array}{l}\text { Discours } \\
\text { d'accompagne- } \\
\text { ment (politiques, } \\
\text { administratifs, } \\
\text { commerciaux, } \\
\text { médiatiques...) }\end{array}$ & $\begin{array}{l}\text { Sociologie, } \\
\text { sciences } \\
\text { économiques, } \\
\text { politiques, de } \\
\text { l'information et } \\
\text { de la } \\
\text { communication }\end{array}$ & $\begin{array}{l}\text { Quelle insertion } \\
\text { de dispositifs en } \\
\text { ligne dans les } \\
\text { systèmes pré- } \\
\text { existants? } \\
\text { Quel futur } \\
\text { acteur social } \\
\text { former? } \\
\text { Quels } \\
\text { investissements } \\
\text { pour quels } \\
\text { profits? }\end{array}$ \\
\hline Méso & $\begin{array}{l}\text { Formation/ } \\
\text { classe } \\
\text { Relation des } \\
\text { cours entre } \\
\text { eux dans une } \\
\text { même } \\
\text { formation }\end{array}$ & $\begin{array}{l}\text { Discours des } \\
\text { cadres de } \\
\text { l'enseignement } \\
\text { (inspecteurs, } \\
\text { directeurs, } \\
\text { conseillers } \\
\text { pédagogiques...) } \\
\text { Discours des } \\
\text { formateurs, } \\
\text { coordonateurs }\end{array}$ & $\begin{array}{l}\text { Sciences de } \\
\text { l'éducation, de } \\
\text { l'information et } \\
\text { de la } \\
\text { communication }\end{array}$ & $\begin{array}{l}\text { Quel dispositif } \\
\text { pour quel } \\
\text { contexte? } \\
\text { Quelles } \\
\text { compétences } \\
\text { professionnelles } \\
\text { pour les } \\
\text { enseignants? } \\
\text { Quelle } \\
\text { articulation dans } \\
\text { le curriculum? }\end{array}$ \\
\hline Micro & $\begin{array}{l}\text { Classe/ } \\
\text { individus } \\
\text { relation } \\
\text { enseignant- } \\
\text { apprenants et } \\
\text { apprenants- } \\
\text { apprenants } \\
\text { Construction } \\
\text { du savoir }\end{array}$ & $\begin{array}{l}\text { Interactions } \\
\text { enseignant- } \\
\text { apprenant } \\
\text { et apprenants- } \\
\text { apprenants }\end{array}$ & $\begin{array}{l}\text { Psychologie, } \\
\text { Ethologie, } \\
\text { Neuro- } \\
\text { sciences }\end{array}$ & $\begin{array}{l}\text { Comment } \\
\text { enseigner et } \\
\text { apprendre de } \\
\text { façon efficace } \\
\text { dans un dispositif } \\
\text { multimodal } \\
\text { synchrone? }\end{array}$ \\
\hline
\end{tabular}

Les limites de cet article ne nous autorisant pas à développer les trois niveaux de recherche sus mentionnés, nous nous concentrerons dans la suite de notre propos sur les niveaux méso et micro mais soulignons l'importance 
qu'il y a à s'intéresser aux politiques économico-institutionnelles qui configurent en quelque sorte les deux niveaux (méso et micro) du « terrain » et de ses acteurs.

Il est en effet important d'être conscient des enjeux économicoindustriels qui surdéterminent les incitations, les pressions voire les décisions d'équipements en matériel technique d'institutions scolaires de tous niveaux.

La plupart du temps en Sciences du langage, on ne parle pas de données mais d'un corpus de communication pédagogique en ligne (que l'on construit en fonction d'une question de recherche) qui peut être étudié sous différents angles méthodologiques parmi lesquels :



Ces quatre niveaux d'analyse se combinent et peuvent venir apporter des réponses complémentaires à des questions telles que : Comment enseigne-ton dans ces nouveaux dispositifs en ligne ? Quel ethos d'enseignant se développe dans ces environnements? Quelles nouvelles pratiques sont en émergence? Nous donnerons rapidement des exemples de résultats provenant de chacune de ces quatre perspectives de recherche en Sciences du langage. Précisons que ces quatre perspectives ne sont pas les seules, 
qu'elles sont données à titre d'exemple tout comme le sont les questions posées (bien entendu innombrables dans chacune de ces perspectives).

\section{L'analyse des interactions}

Pour ne prendre que l'exemple concernant les recherches menées autour de l'acquisition des langues, elles s'inscrivent dans le prolongement des recherches interactionnistes (De Pietro, Matthey, \& Py, 1989, Long, 1991 \&, Dausendschön-Gay, Krafft,1993,) qui ont mis en évidence le rôle constitutif de l'interaction pour les processus de l'apprentissage et plus particulièrement des interactions où les interlocuteurs focalisent leur attention sur la forme. Ces focalisations se concrétisent à travers les séquences potentiellement acquisitionnelles et représentent des traces des opérations cognitives qui interviennent dans la construction de l'interlangue de l'apprenant (De Pietro, Matthey \& Py, 1989).

En partant de l'idée que les interactions électroniques ont des similitudes avec celles des discussions en classe, Pellettieri (2000), Smith (2003), Nicolaev (2010) transposent les modèles d'analyse interactionnelle en classe de langue à l'analyse des interactions synchrones en ligne. Ils montrent que ces dernières sont susceptibles de déclencher des séquences propices à l'acquisition similaires à celles qui existent en présentiel. Dans la même ligne, Nicolaev dans sa thèse de doctorat (en cours), étudie comment les différentes modalités de communication (orale, écrite, visuelle) dans un dispositif synchrone vidéographique sont investies pour exprimer et élucider un problème de communication. Les premiers résultats de son analyse montrent que la multimodalité a un impact positif dans l'expression et l'élucidation d'un obstacle linguistique. Ainsi, lorsqu'un apprenant indique un problème de langue par un regard interrogatif ou par des gestes, l'image retransmise par la webcam permet aux apprentis-tuteurs de le repérer et de l'éclaircir. La modalité visuelle permet aux tuteurs d'étayer les explications à l'aide des gestes illustratifs. Quant au clavardage, il permet aux tuteurs d'appuyer leurs explications orales et offre à l'apprenant la possibilité de visualiser l'information, en facilitant ainsi l'accès au sens du discours.

\section{L'analyse sociolinguistique}

Ce type d'analyse permet par exemple de s'interroger sur les spécificités de la langue française enseignée. Dans un précédent article (Develotte, \& Mangenot, 2007), nous avions cherché à mettre en évidence, les variantes de la langue qui étaient enseignées par le truchement du F1L asynchrone au cours de ses quatre premières années d'existence. Je les résume rapidement :

Au niveau verbal tout d'abord, on constatait des variations par rapport à la norme (en matière d'enseignement du français) qui s'exprimait en termes de choix par les étudiants de ressources exposant à des accents étrangers (canadienne, marocain, cap verdienne, vietnamien) ou régional (bisontin, alsacien, martiniquais), à des intonations marquées (banlieue, « jeune») à un lexique renvoyant à la langue des jeunes (« je suis dix fois 
plus adepte », « les Américains, ils ont pas tilté », « c'est fort quoi ») et même à un échantillon de verlan.

$\mathrm{Au}$ niveau du non verbal, dans la forme synchrone, ces descriptions restent valides, on peut toutefois y ajouter l'emploi d'abréviations utilisées lors l'usage du chat et les formes d'expressions non verbales qui sont transmises par l'image de la webcam (par exemple tous les gestes associés à l'interaction ou les gestes illustratifs).

\section{L'éthologie de la communication}

À la suite de Goffman (1973) qui prônait une «méthodologie de l'interaction », l'approche éthologique, est «naturaliste» avec en conséquence un effort de description globale des phénomènes aussi bien verbaux que "posturo-mimo-gestuels » dans leurs rapports à l'interaction duelle, et "compréhensive" dans la mesure où elle relie les faits observés au cadre technique et institutionnel et aux commentaires des sujets interviewés en post-enregistrements. Dans de récents travaux, nous nous sommes intéressés, Jacques Cosnier et moi-même (2011) à la conversation en face à face en ligne. C'est cette même approche qui a été reprise pour identifier la gamme de comportements de tuteurs en ligne dans l'utilisation de la multimodalité permise par la visioconférence poste à poste pour une tâche donnée (Develotte, Guichon, \& Vincent, 2010). Les résultats font état d'une gradation dans la façon de se servir sur Skype de la visio ${ }^{21}$ par rapport aux autres modalités (écrit et oral seul) selon l'utilité perçue de la visio pour la conduite du tutorat et selon leur capacité à gérer différents espaces de travail. Nous avons mis en évidence qu'il y a différents degrés d'investissement de la visio et que celle-ci peut être utilisée soit pour être vue par les apprenants, soit pour voir les apprenants. Les informations apportées par l'image visio du tuteur vont être d'ordre psychopédagogique (le feedback apporté), d'ordre culturel (les éléments culturels propres à un natif de la langue-cible) ou bien d'ordre linguistique (un geste vient compléter une information donnée à l'oral). Quand la visio est utilisée, celleci joue un rôle majeur dans la dimension socio-affective de la communication pédagogique et dans la construction de la relation interpersonnelle. La visio permet de concrétiser la présence à distance, d'installer une connivence manifeste entre les participants et, partant, d'intensifier la qualité de la relation pédagogique comme les entretiens le soulignent.

\section{L'analyse du discours multimédia}

Les analyses relevant de ce niveau cherchent à mettre en relation la «matérialité » de la forme de communication avec les productions discursives qu'elle peut accueillir, autrement dit, l'influence du dispositif énonciatif sur les interactions. Afin d'expliciter quelles libertés et quelles contraintes d'expression détient la communication sur un forum ou une

${ }^{21}$ L'image « visio » désigne l'image des locuteurs qui apparaît dans les fenêtres d'un environnement tel que Skype (ceci pour la différencier de l'image « vidéo » qui peut être celle d'un clip présenté à l'écran comme document déclencheur d'une activité). 
plate-forme synchrone, j'ai proposé l'opposition «espace d'exposition discursive » et «espace de production discursive»(Develotte, 2006b). Il s'agit d'étudier les différents niveaux enchâssés les uns dans les autres que sont la mise en écran, la mise en média, la mise en rubriques, la mise en discours. Selon que le mode de communication peut être multimodal ou non (images, vidéos, texte, fichiers son, audio synchrone), selon la taille de la fenêtre d'écriture etc., les possibilités de communication seront différentes et l'expression elle-même variera (forme écrite du français abrégée pour les chats, utilisation d'émoticônes). Dans les premières analyses que j'avais effectuées à partir de ce cadre j'avais mis en évidence la prévalence dans le forum étudié de la logique technique sur celle de l'usager néophyte (à travers un espace de communication inutilement compliqué), signe visible de l'énonciation informatique derrière l'écran. Cette perspective renvoie aux travaux d'Emmanuel Souchier sur l'énonciation éditoriale « le texte considéré comme objet concret et qui a été configuré à travers cette activité plurielle qu'est l'énonciation éditoriale. » (1999 : 145). Si l'on cherche à appliquer cette optique à la communication en ligne reviennent de plein droit les questions relatives à l'idéologie qui s'inscrit dans l'apparente neutralité des formes non plus du papier mais de l'écran. On rejoint ici les centres d'intérêt classiquement issus de l'analyse du discours et c'est en ce sens que l'analyse du discours multimédia se situe à un autre niveau que celui de l'analyse des interactions, dans la mesure où elle débouche sur les choix idéologiques, culturels, historiques d'une société (il vaudrait d'ailleurs mieux parler aujourd'hui d'un monde) qui transcendent et informent la situation de communication. Si l'on compare ce niveau d'analyse avec celui précédemment décrit pour l'analyse des interactions, on peut dire que l'on cherche ici à travailler l'articulation entre les niveaux méso et macro.

\section{Quelques changements sur le plan des recherches}

Les quelques illustrations des recherches ci-dessus montrent que la dimension numérique a, en tout état de cause, été prise en compte et intégrée dans les protocoles d'étude des nouveaux corpus en ligne. Sur le plan des recherches en didactique des langues, l'un des changements les plus notables introduit par le numérique réside dans la quantité des données susceptibles d'être prises en compte, par exemple la quasi intégralité d'une formation. C'est en effet un cas inédit dans les situations d'enseignement que la totalité des échanges sur une plate-forme puisse être conservée. C'est à de tels corpus (soit quelques 3200 messages enseignants-apprenants et apprenants-apprenants, Combe-Celik, 2010) que s'attellent les jeunes chercheurs pour décrire la communication pédagogique en ligne.

Au niveau de la méthodologie de la recherche, si les recherches sur l'oral se sont développées grâce à l'arrivée du magnétophone (Rivenc, 2006) les recherches sur la communication en ligne se développent de plus en plus actuellement à travers l'utilisation de logiciels d'enregistrement de captures d'écran (Sreenvideorecorder, Camtasia) et de traitements des données permettant par exemple de dissocier les différentes modalités textuelles, orales et gestuelles dans la communication par visioconférence poste à poste (Transana, Elan). 


\section{Conclusion}

Dans ce qui précède j'ai cherché à montrer que différents indices incitent à dire que l'on assiste aux réalisations de « la nouvelle donne » dont parlait Daniel Coste et dont il annonçait avec justesse les éléments qui se sont confirmés depuis 10 ans : «Désormais on est entré dans une logique de réseaux où les émetteurs sont multiples et où les destinataires apprenants ont de plus en plus loisir de se connecter à des sources plurielles, à interagir entre eux et, singulièrement pour ce qui est de leur compétence langagière, à affronter de nombreuses instances d'évaluation, formelles ou non, officielles ou non. » (1999: 10)

D'un côté, du côté du monde social, « les consommateurs d'aujourd'hui sont des utilisateurs, plus actifs et productifs que les consommateurs de l'économie industrielle de l'information. Ce changement remodèle les relations nécessaires à la réussite des entreprises, impliquant une intégration plus étroite des utilisateurs dans le processus de production (...). Il nécessite des talents de leadership et des objectifs différents. » (Benkler : 180). La participation plus active des internautes a suscité la création de néologismes tels que les "consomacteurs", « aucteurs » mettant l'accent sur la démarche participative de l'internaute. De l'autre, du côté de l'enseignement /apprentissage des langues quelle place les "apprenacteurs" viendront-ils prendre?

Les projets comme Le français en première ligne, comme beaucoup d'autres s'inscrivent dans cette logique de réseaux, de mise en relation de locuteurs, d'ouverture de la classe vers des évaluations sociales autant que scolaires ou universitaires. C'est dans cet esprit qu'avec le soutien de l'AUF $^{22}$ j'ai cherché à diffuser ce projet dans le mondeafin de mettre en contact des classes de futurs enseignants avec des classes d'apprenants de français :

- la première raison, c'est qu'à la différence des manuels de langue qui se doivent d'être universalistes pour des raisons commerciales évidentes, les projets qui mettent en contact les classes entre elles permettent de contextualiser les enseignements selon les besoins des cultures d'enseignement et d'apprentissage, d'ajuster une offre de formation à une demande non seulement linguistique mais aussi culturelle.

- la deuxième raison, c'est qu'actuellement, nous assistons à une coexistence de la tendance à la privatisation des connaissances avec celle de la liberté d'accès et de la libre diffusion des connaissances (ainsi, de nombreux cours d'universités prestigieuses sont en ligne, comme ceux du MIT). Cette situation est-elle amenée à perdurer dans le futur? Quel sera le poids des uns et des autres dans le développement de liens éducatifs mondialisés ? Les évolutions du domaine éducatif dépendent des politiques publiques mais aussi de la volonté des acteurs et de leurs besoins d'échanger. Sous les stratégies des institutions, de Certeau (1990) a bien montré que les tactiques des acteurs déjouaient les parcours tracés et s'insinuaient dans les interstices de liberté qu'ils ne manquaient pas d'y trouver. Il semble important que les

${ }^{22}$ http://www.aidenligne-francais-universite.auf.org/spip.php?page=sommaire_appui_ens_fr 
solidarités militantes de l'éducation relayée par les associations du champ occupent le terrain en le dessinant à l'image de ce qu'elles souhaitent qu'il soit.

La didactique des langues a connu d'autres changements, plus inattendus (Cortier, Parpette, 2006) qui ont eu des conséquences sans doute plus radicales que celles introduites par le numérique dans les pratiques aujourd'hui. La révolution de l'enseignement du français engendrée par la publication du Français fondamental était ainsi d'une autre ampleur comme le rappelle Anne-Marie Chartier :

« Les 67 pages du Français fondamental, petite vague devenue tsunami, ébranlent tout l'édifice scolaire de l'enseignement du français (langue maternelle ou seconde), dont la pierre angulaire, scellée au début du XXe siècle, était l'étude des textes français. » (2009: 141) puisqu'il a fait basculer de l'enseignement de la langue par les textes littéraires à celui par l'étude de l'interaction orale. Il n'empêche que l'on sent des frémissements de changements dans les pratiques d'enseignement, d'apprentissage et de recherche associées à l'intrusion du numérique ces dernières années mais ce sera seulement a posteriori que l'on pourra juger de la hauteur de la vague numérique sur laquelle la didactique des langues apprend à surfer aujourd'hui.

\section{Christine DEVELOTTE, ICAR, INRP, Lyon.}

\section{Bibliographie}

Barbot, M-J., 1997 « Cap sur l'autoformation : multimédias des outils à s'approprier », in "Multimédia réseaux et formation», $\mathrm{n}^{\circ}$ spécial du Français dans le monde recherches et applications, juillet 1997, p. 54-63.

Benkler, Y, 2009, La richesse des réseaux Marchés et libertés à l'heure du partage social, Lyon, Presses universitaires de Lyon (traduction de The Wealth of Networks : How Social Production Transforms Markets and Freedom, Yale University Press, 2006, en ligne sur le site: www.benkler.org/Benkler Wealth Of Networks.pdf

Cadre européen commun de référence, 2000, Division des Politiques Linguistiques, Strasbourg, Conseil de l'Europe http://www.coe.int/t/dg4/linguistic/source/framework_FR.pdf

Castells, M., 2001, L'Ere de l'information, tome 1: La Société en réseaux, $2^{\mathrm{e}}$ edition de la traduction française, Paris, Fayard.

Combe-Celik, C. 2010, "Pratiques discursives dans une formation en ligne à la didactique du français langue étrangère », thèse de doctorat en sciences du langage, université Stendhal Grenoble 3.

Cortier, C. Parpette, C. (dir) 2006 «De quelques enjeux et usages historiques du français fondamental », Documents pour l'histoire du français langue étrangère ou seconde, $\mathrm{n}^{\circ} 36$, juin.

Cosnier, J., Develotte, C., 2011, "Ethologie compréhensive de la conversation en visioconférence poste à poste » in Develotte, C, Kern, R., Lamy, M-N., Décrire la conversation en ligne, Lyon, ENS Editions. 
Chartier, A. M. 2009.« Note critique» sur Cortier, C., Parpette, C., (dir) 2006 « De quelques enjeux et usages historiques du français fondamental », Histoire de l'éducation, 2009/2, n 122, p. 136-142.

Dausendschön-Gay, U. \& Krafft, U. (1993). "La séquence analytique". Bulletin CILA, 57, 137-157.

Debray, R., 1979 Le pouvoir intellectuel en France, Ramsay.

De Pietro, J.-F., Matthey, M. \& Py, B. (1989). "Acquisition et contrat didactique: les séquences potentiellement acquisitionnelles de la conversation exolingue". In D. Weil \& H. Fugier (Eds),

Martinez P \& Pekarek S. 2000 La notion de contact de langues en didactique, Notions en Questions n ${ }^{\circ} 4$, ENS Editions.

Develotte, C. 1997, «Lecture et cyberlecture » in « Multimédia réseaux et formation", $\mathrm{n}^{\circ}$ spécial du Français dans le monde recherches et applications, juillet 1997, p. 94-104.

Develotte, C., (1998) « Nouvelles technologies / nouveaux discours dans l'enseignement / apprentissage des langues ", Etudes de linguistique appliquée. $\mathrm{N}^{\circ} 112$, pp. 421-434. Version de l'auteur disponible sur Internet : [ http://halshs.archives-ouvertes.fr/halshs-00151856 ]

Develotte, C., (2003). "Accès aux ressources documentaires en situation d'autonomie : pratiques et représentations », Alsic, Vol. 6, ${ }^{\circ} 1$, http://alsic.u-strasbg.fr/Num10/develotte/alsic_n10-rec1.pdf

Develotte, C., (2006) "L'étudiant en autonomie et ses professeurs virtuels : comment se personnalise la communication en ligne ?», Synergies Chili, $\mathrm{N}^{\circ}$ 2, version de l'auteur disponible sur Internet : [ http://halshs.archives-ouvertes.fr/halshs-00151852 ]

Develotte, C., (2006b). " Décrire l'espace d'exposition discursive dans un campus numérique ». Le Français dans le monde. Recherches et applications, pp. 88-100. Version de l'auteur disponible sur Internet : [ http://halshs.archives-ouvertes.fr/halshs-00151851 ]

Develotte, C., Kern, R., Lamy, M.-N., 2011, Décrire la conversation en ligne, le face à face distanciel, ENS Editions.

Develotte, C., Guichon, N., Kern, R., (2008) «"Allo Berkeley? Ici Lyon... Vous nous voyez bien?" Étude d'un dispositif d'enseignementapprentissage en ligne synchrone franco-américain », ALSIC, http://halshs.archives-ouvertes.fr/halshs-00346486/fr/

Develotte, C., Mangenot, F., Zourou, K., (2005) « Situated creation of multimedia activities for distance learners : motivational and cultural issues ", $\operatorname{Re} C A L L, \mathrm{~N}^{\circ} 17$, pp. 229-244. Version des auteurs disponible sur Internet : [ http://halshs.archives-ouvertes.fr/halshs-00151847

Develotte, C., Guichon, N., Vincent, C., (2010a) "The use of the webcam for teaching a foreign language in a desktop videoconferencing environment" $\operatorname{ReCALL}(23,3)$.

Develotte, C., Mangenot, F. (2010b) « Former aux tutorats synchrone et asynchrone en langues », Distance et savoirs, vol. 8, n³, p. 345-359.

Guichon, N, Drissi, S., 2008, «Tutorat de langue par visioconférence : comment former aux régulations pédagogiques?», Les Cahiers de l'Acedle, volume 5, numéro 1, Recherches en didactique des langues, http://acedle.org/spip.php?article1021

Guichon, N. (à paraître) L'intégration des technologies dans l'enseignement des langues, Paris : Didier.

Jones, R.2004, The Problem of Context in Computer Mediated 
Communication. In Levine, P. \& Scollon, R. (eds), Discourse \& Technology multimodal discourse analysis. Washington DC: Georgetown University Press, 20-33.

Krikorian, G. \& Kapczynski, A., Access to Knowledge in the Age of Intellectual Property, Zone Books, the MIT Press, en ligne sur le site : http://mitpress.mit.edu/catalog/item/default.asp?ttype=2\&tid=12589

Lankshear, C. (2003). "The Challenge of digital Epistemologies ", Education, Communication and Information 3(2), pp. 167-186.

Long, M. H. (1991). "Focus on form: A design feature in language teaching methodology". In de Bot, K., Ginsberg, R. B., \& Kramsch, C. (dir). Foreign language research in cross-cultural perspective. pp. 39-52. Amsterdam : John Benjamins.

Mogi, R., 2010, « Nouveau métier, nouvelle formation du conseiller en ligne en didactique des langues et des cultures étrangères », doctorat en sciences du langage, université Lille 3.

Nicolaev, V. (2010). "Les négociations de sens dans un dispositif d'apprentissage des langues en ligne synchrone par visioconférence". Les Cahiers de l'Acedle, vol. 7, n², pp. 169-198.

O'Rourke, B., «Form-focused Interaction in Online Tandem Learning », Calico Journal, vol.22, n³, 2005, p. 433-466.

Pellettieri, J. (2000). "Negotiation in Cyberspace - the Role of Chatting in the Development of Grammatical Competence". In Warschauer, M. \& Kern R. (dir.). Network-based LT : Concepts and Practice. Cambridge : CUP.

Pisani, F., Piotet, D., 2008, Comment le web change le monde L'alchimie des multitudes, Pearson Education France.

Poyet, F., Develotte, C., 2011, L'éducation à l'heure du numérique. État des lieux, enjeux et perspectives, Lyon, ENS de Lyon-INRP.

Rivenc, P. «Les auteurs du Français fondamental face à un objet nouveau et insolite : l'interaction orale », in Cortier, C. Parpette, C. (dir) 2006 « De quelques enjeux et usages historiques du français fondamental », Documents pour l'histoire du français langue étrangère ou seconde, $\mathrm{n}^{\circ} 36$, p. 35-49.

Slodzian, M., 2000, « Contacts de langues, technologies et transfert des connaissances ", La notion de contact de langues en didactique, Notion en Question $\mathrm{n}^{\circ} 4$, ENS Editions.

Smith, B. (2003). "Computer-mediated negotiated interaction: An expanded model". Modern Language Journal, vol. 87, $\mathrm{n}^{\circ}$ 1. pp. 38-57.

Vanderplank R., 2010, "Déjà vu ? A decade of research on language laboratories, television and video in language learning ", Language Teaching, $43: 1$, p. 1-37. 